\title{
INFORMATION AND COMPUTATION
}

Essays on Scientific and Philosophical Understanding of Foundations of Information and Computation 


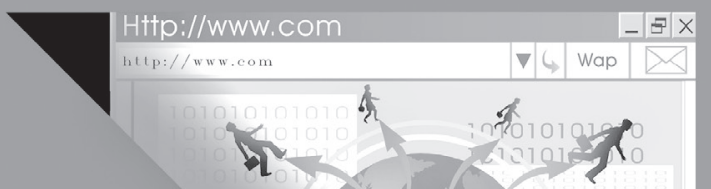

World Scientific Series in Information Studies - Vol. 2

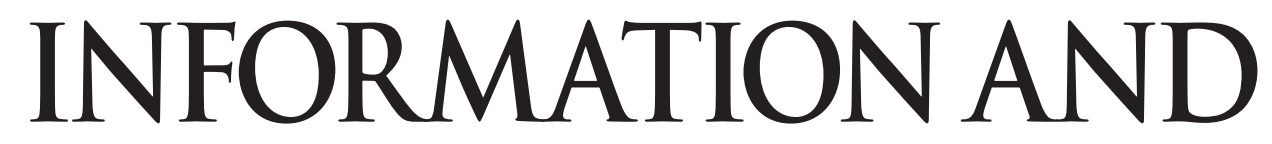

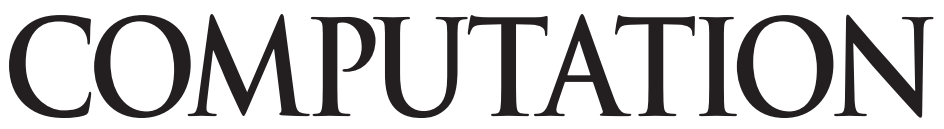

Essays on Scientific and Philosophical Understanding of Foundations of Information and Computation

\section{Gordana Dodig-Crnkovic}

Mälardalen University, Sweden

\section{Mark Burgin}

University of California, Los Angeles, USA 


\section{World Scientific Series in Information Studies}

(ISSN: 1793-7876)

Series Editor: Mark Burgin (University of California, Los Angeles, USA)

\section{International Advisory Board:}

Søren Brier (Copenhagen Business School, Copenhagen, Denmark) Tony Bryant (Leeds Metropolitan University, Leeds, United Kingdom)

Gordana Dodig-Crnkovic (Mälardalen University, Eskilstuna, Sweden)

Wolfgang Hofkirchner (ICT\&S Center, University of Salzburg, Salzburg, Austria)

William R King (University of Pittsburgh, Pittsburgh, USA)

Vol. 1 Theory of Information - Fundamentality, Diversity and Unification by Mark Burgin

Vol. 2 Information and Computation - Essays on Scientific and Philosophical Understanding of Foundations of Information and Computation edited by Gordana Dodig-Crnkovic \& Mark Burgin 


\section{Published by}

World Scientific Publishing Co. Pte. Ltd.

5 Toh Tuck Link, Singapore 596224

USA office: 27 Warren Street, Suite 401-402, Hackensack, NJ 07601

UK office: 57 Shelton Street, Covent Garden, London WC2H 9HE

\section{British Library Cataloguing-in-Publication Data}

A catalogue record for this book is available from the British Library.

\section{World Scientific Series in Information Studies - Vol. 2 INFORMATION AND COMPUTATION Essays on Scientific and Philosophical Understanding of Foundations of Information and Computaiton}

Copyright $(0) 2011$ by World Scientific Publishing Co. Pte. Ltd.

All rights reserved. This book, or parts thereof, may not be reproduced in any form or by any means, electronic or mechanical, including photocopying, recording or any information storage and retrieval system now known or to be invented, without written permission from the Publisher.

For photocopying of material in this volume, please pay a copying fee through the Copyright Clearance Center, Inc., 222 Rosewood Drive, Danvers, MA 01923, USA. In this case permission to photocopy is not required from the publisher.

ISBN-13 978-981-4295-47-5

ISBN-10 981-4295-47-7

Printed in Singapore. 


\section{CONTENTS}

Preface vii

1. Cybersemiotics and the Question of Knowledge 1 Søren Brier

2. Information Dynamics in a Categorical Setting 35 Mark Burgin

3. Mathematics as a Biological Process 79 G. J. Chaitin

4. Information, Causation and Computation 89 John Collier

5. From Descartes to Turing: The Computational Content of Supervenience

S. Barry Cooper

6. A Dialogue Concerning Two World Systems: Info-Computational vs. Mechanistic

Gordana Dodig-Crnkovic and Vincent C. Müller

7. Does Computing Embrace Self-Organization?

Wolfgang Hofkirchner

8. Analysis of Information and Computation in Physics Explains

Cognitive Paradigms: From Full Cognition to Laplace

Determinism to Statistical Determinism to Modern Approach

Vladik Kreinovich, Roberto Araiza and Juan Ferret 
9. Bodies-Both Informed and Transformed Embodied Computation and Information Processing Bruce J. MacLennan

10. Computation on Information, Meaning and Representations: An Evolutionary Approach

Christophe Menant

11. Interior Grounding, Reflection, and Self-Consciousness Marvin Minsky

12. A Molecular Dynamic Network: Minimal Properties and Evolutionary Implications Walter Riofrio

13. Super-recursive Features of Evolutionary Processes and the Models for Computational Evolution Darko Roglic

14. Towards a Modeling View of Computing Oron Shagrir

15. What's Information, for an Organism or Intelligent Machine? How can a Machine or Organism Mean?

Aaron Sloman

16. Inconsistent Knowledge as a Natural Phenomenon: The Ranking of Reasonable Inferences as a Computational Approach to Naturally Inconsistent (Legal) Theories Kees (C.N.J.) de Vey Mestdagh \& Jaap Henk (J.H.) Hoepman

17. On the Algorithmic Nature of the World 


\section{PREFACE}

\section{INFORMATION AND COMPUTAITON - OMNI PRESENT AND PERVASIVE}

The world is full of information. Information is everywhere. It is in us and outside us. Information connects us to other people and it separates and alienates us. Information makes us happy and sad. It brings joy, satisfaction, grief, delight, pain, and consolation. In order for anything to exist for an individual, the individual must get information on it, either by means of perception or by re-organization of the existing information into new patterns and networks in the brain. With the advent of World Wide Web and a prospect of semantic web, the ways of information supply for individuals, networks of humans and machines and for humanity as a whole are becoming strategically important in a number of ways. Information becomes pivotal for communication, research, education systems, government, businesses and basic functioning of everyday life. Information architecture can rightly be characterized as the backbone of our civilization, as our essential supporting mechanism.

Information has developed into the leading factor in the world's economy as the following quotation demonstrates:

"Today, we need to look at what investments - and investment philosophies - will position us for success in the 21 st century, when information, not manufacturing, will be the spark and engine of economic leadership.

The forces we face are massive - a global recession, climate change, an explosion of information that is reshaping how we conduct our business and personal lives. I believe that IT can transform these increasingly intersecting challenges into opportunities." (Banerjee, 2009).

Hope is put into information technology development such as the cloud - the next stage of the internet - a huge, intelligent, sustainable 
infrastructure where everything will be delivered as services: computing power, business processes and personal interactions.

However, in spite of all strategic importance and omnipresence of information, scientists have not come to the decisive conclusion about the essence and nature of information. There are hundreds of opinions and dozens of theories (cf., for example (Adriaans and van Benthem, 2008; Burgin, 2010)), but still the information concept is evasive and vague although correct knowledge about information is necessary for our understanding of the world we live in.

Information is related to everything and every thing is related to information. However, the most intimate relations exist between information and energy. On the one hand, principles of the general theory of information developed in (Burgin, 2003; 2005a; 2010) demonstrate that energy is a kind of information in the broad sense. This correlates with the von Weizsäcker's idea (1995/2006) that energy might in the end turn out to be information. In essence, all physical characteristics become different kinds of information obtained about physical systems and processes. According to one of the outstanding physicists of the $20^{\text {th }}$ century John Archibald Wheeler, it means that every physical quantity derives its ultimate significance and meaning from information (Wheeler, 1990; 1994).

The most fundamental approach to physics based on information, the, so-called, $u r$-theory, has been developed in the school of von Weizsäcker (von Weizsäcker, 1958; von Weizsäcker, Scheibe and Süssmann, 1958; Castell, et al, 1975-1986; Lyre, 2002). The main idea is that what physicists learn about nature comes from observation and experiments through measurement. According to von Weizsäcker, measurement is information extraction from physical objects and processes. Thus, physical objects and processes are entirely characterized by the information that can be acquired from them. In such a way, physics reduces to predicting measurement outcomes.

Frieden (1998) also developed a new approach to physics based on Fisher information, explaining that physics as a knowledge system about the universe is built from knowledge acquired through information reception and processing. According to this conception, the observer is included into the phenomenon of measurement as his properties as an 
information receiver influence what data are collected and how they are collected and interpreted. The basic contention of Frieden is that physicists think that they study material systems as they are, while they actually study information that they are able to get from these systems.

On the other hand, according to the general theory of information, information is energy in the structural world, e.g., in the world of knowledge and data, while conventional energy is energy in the physical world, i.e., the world of material objects. At the physical level of reality, relations between information and energy are considered by Umpleby (2007) who comes to a conclusion that at the level of data signal processing "difference" is more elementary than information. Information is what the universe is, in some sense, made of, while the difference is a trait in a structure of a cognitive agent.

A grounded analysis of this statement by some researchers shows that actually any difference is accessible only through information reception. Consequently, information is a more basic (and in some sense, more elementary) phenomenon than "difference."

However, many other researchers believe that data are basic elements of which information is made, and information is stuff of which knowledge is made. This approach finds its structural representation in the, so-called, Data-Information-Knowledge Pyramid.

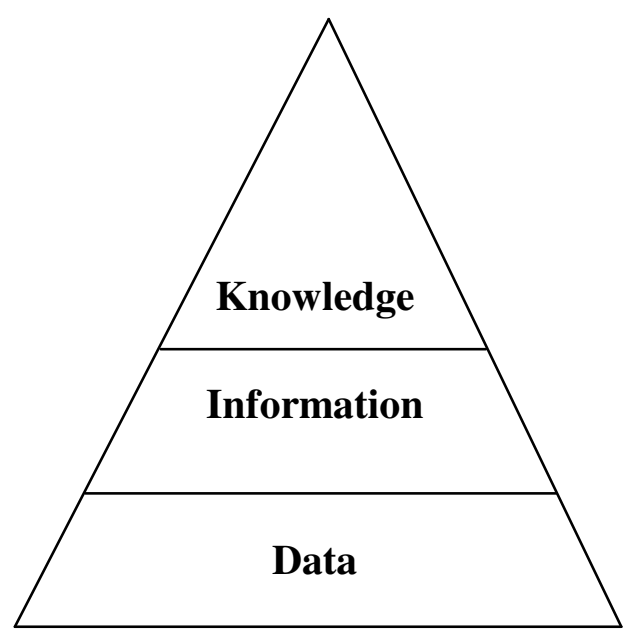

Fig. 1. The Data-Information-Knowledge (DIK) Pyramid 
There are different interpretations of the levels from this pyramid (cf., for example, (Stenmark, 2002; Boisot and Canals, 2004; Burgin, 2010)). The most popular of them tells:

Information is structuring of data (or structured data)

Knowledge is structuring of information (or structured information)

However, another approach (cf., for example, (Meadow and Yuan, 1997)) suggests a different picture:

Data usually means a set of symbols with little or no meaning to a recipient.

Information is a set of symbols that does have meaning or significance to their recipient.

Knowledge is the accumulation and integration of information received and processed by a recipient.

This and many other definitions show absence of consensus on the meaning of these terms. For instance, in many books and papers, the terms knowledge and information are used interchangeably, even though the two entities, being intertwined and interrelated concepts, are far from identical. While some researchers define knowledge and/or data in terms of information, others define information in terms of data and knowledge. For instance, Kogut and Zander (1992) conceive information as "knowledge which can be transmitted without loss of integrity," while Tuomi (1999) argues that data emerge as a result of adding value to information.

In a similar way, commonplace usage of words data and information blurs differences between these concepts. For instance, Machlup and Mansfield write (1983):

"Data are the things given to the analyst, investigator, or problemsolver; they may be numbers, words, sentences, records, assumptions just anything given, no matter in what form and of what origin... Many writers prefer to see data themselves as a type of information, while others want information to be a type of data."

All these and many other inconsistencies related to the DataInformation-Knowledge Pyramid cause grounded criticism of this approach to understanding data, knowledge, and information. For instance, Capurro and Hjorland write (2003) that the semantic concept of information, located between data and knowledge is not consistent with 
the view that equates modern information management with information technology. Boisot and Canals (2004) criticize distinctions that have been drawn between data, information, and knowledge by those who analyzed the Data-Information-Knowledge Pyramid. Fricke (2008) also offers valid arguments that the hierarchy is unsound and methodologically undesirable. In addition, researchers criticized various implications of the Data-Information-Knowledge Pyramid. For instance, Tuomi (1999) argues that in contrast to the conventional estimation that knowledge is more valuable than information, while information is superior to data, these relations have to be reversed.

Problems with the Data-Information-Knowledge Pyramid are resolved in the general theory of information where relations between data, information and knowledge are represented by the KnowledgeInformation-Matter-Energy (KIME) Square.

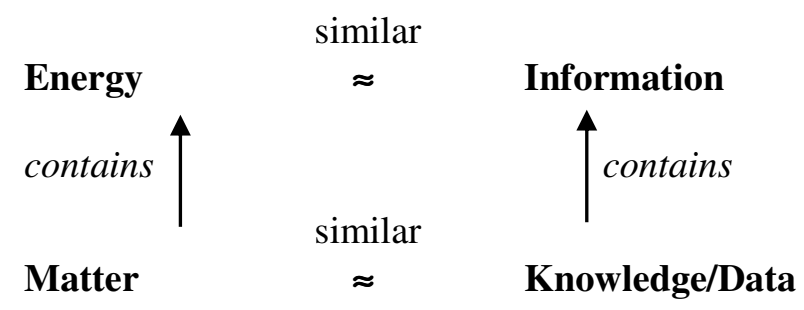

Fig. 2. The Knowledge-Information-Matter-Energy (KIME) Square

The KIME Square visualizes and embodies the following principle:

\section{Information is related to knowledge and data as energy is related to matter.}

It means that knowledge and data contain information as matter (material bodies) contains energy. As it is possible to convert matter into energy, extract energy from material bodies and convert energy into material things, e.g., into subatomic particles, it is doable to convert knowledge and data into information and information into knowledge and data. When people are learning, they receive information and then transform it into knowledge and data (Burgin, 2001; 2010). As a result we come to a clear understanding of roles that teachers and student play in the educational process: a teacher cannot put knowledge into the heads 
of her students, she is able only to provide information and students themselves have to convert this information into knowledge.

In addition, the KIME Square tells us that while data and knowledge belong to the same type of objects, the essence of information is very different (Burgin, 2005a). Like energy gives dynamics to substance, information produces dynamics of data and knowledge.

Some may be confused that data and knowledge are considered as similar entities. We can explain their relations in a metaphorical way. The first metaphor elucidates that data and knowledge are like different types of molecules: data are like molecules of water, which has two atoms, while knowledge is like molecules of DNA, which contains billions of atoms. One more metaphor provides another analogy: data and knowledge are like living beings, but data are like bacteria, while knowledge is like a human being.

In addition, Umpleby argues that information can be directly related to matter and energy while adopting Bateson's statement that information is the difference that makes a difference (Bateson, 1972). Taking this statement as a candidate for a definition of information, some researchers conceive that it would be as deceptive as it is beautiful. First, without sufficiently exact definition of difference, this not a definition as it is wrong to define one unknown term through another unknown term. This is a logical fallacy. Second, even if we take the mundane meaning of the word difference, we may come to a conclusion that anything is a difference because anything is different from something else. Besides, being different, any thing makes this difference and thus, we have to logically conclude that anything is information. This makes the concept of information void from a scientific point of view. Fortunately, this is not true and the concept of information is not only meaningful but is becoming one of the pillars of contemporary science.

At the same time, other researchers (Dodig Crnkovic, 2009) believe Bateson's statement reflects the way information functions in physics, cybernetics, biology and number of other fields and take for granted that difference is a clear concept. This belief is based on the assumption that the difference is what a specific cognizing system is capable of detecting as difference. So, the difference is receiver specific. 
In its existence, information is related to many things, such as data, knowledge or difference. It is also intrinsically related to computation. Information is a basic essence of the world, while computation is a process of the dynamic change of information (Dodig Crnkovic, 2006). Indeed, any computation is information processing. However, not any information processing is computation. According to the general theory of information, there are three basic types of information processing (Burgin, 1997; 2010):

- information transmission;

- information transformation;

- information storage.

In information transmission, information changes its place in space.

In information transformation, information itself and/or its representation are changed.

In information storage, information changes its place in time.

There are three reasonable levels of generality in understanding the phenomenon of computation:

On the top level, computation is perceived as any transformation of information and/or information representation.

On the middle level, computation is distinguished as a discrete process of transformation of information and/or information representation.

On the bottom level, computation is recognized as a discrete process of symbolic transformation of information and/or symbolic information representation.

It is necessary to remark that if we do not go beyond the bottom level and insist on discreteness, we would loose continuous time computation realized by general dynamical systems (Bournez, 1999), hybrid systems (Gupta, et al, 1999), and special computing devices, such as the differential analyzer (Shannon, 1941; Moore, 1996).

It looks natural to define computation as transformation of information representation. This is the traditional approach of computationalism (cf., for example, (Kelemen, 2006)). It is also natural to assume that transformations can be discrete, continuous and partially continuous. 
As there are different representations of information, this definition results in separation of substantial types of computations:

1. Symbolic computation when information is represented by symbols.

2. Material computation when information is represented by material things. This can be a biological cell or an atom and can be continuous as there is continuum in many branches of physics.

Artificial devices, such as computers and calculators, perform material computation, representing symbolic computation, which is pivotal. The same process of symbolic computation can be realized by different material computations, e.g., on different computers. Quantum computation is a kind of symbolic computations embodied in material computation where symbols are represented by quantum states.

It is an open problem whether symbolic computation is possible without material computation although the majority of researchers (Dodig-Crnkovic 2009) cannot even imagine such a situation, believing that there is no information without physical representation and there is no computation without information.

At the same time, a broader view of computation reflected by the middle level results in three operational types of computation (Burgin, 2005):

1. Discrete computation when digital or symbolic operations are performed in elementary separated steps.

2. Continuous or analogue computation when operation goes without breaks in time.

3. Piecewise continuous computation, which combines both discrete computation and continuous computation.

In addition, we have three temporal types of computation (Burgin, 2005):

1. Sequential computation, which is performed in linear time.

2. Parallel or branching computation, in which separate steps (operations) are synchronized in time. 
3. Concurrent computation, which does not demand synchronization in time.

Note that while parallel computation is completely synchronized, branching computation is not completely synchronized.

Existence of various types and kinds of computation, as well as a variety of approaches to the concept of computation, shows complexity of understanding computation in the holistic picture of the world.

In this volume, a number of leading researchers and philosophers study problems of information and computation treating information in its dynamical context - time changes of informational structures, their interaction, and impact. From this perspective, we need much better understanding of information processing and its primary form computation than we have now. As there is no information without (physical) representation, the dynamics of information is implemented on different levels of granularity by different physical processes, including the level of computation performed by computing machines and living organisms. There are a lot of open problems related to the nature of information and essence of computation, as well as to their relationships. How is information dynamics represented in computational systems, in machines, as well as in living organisms? Are computers processing only data or information and knowledge as well? How is information processing related to knowledge management and sciences, especially to science of information itself? What do we know of computational processes in machines and living organisms and how these processes are related? What can we learn from natural computational processes that can be useful for information systems and knowledge management? These and similar problems associated with information and computation are treated in the book.

In Chapter 1, "Cybersemiotics and the Question of Knowledge", Søren Brier treats the paninformationalism as a kind of informational structural realism, according to which the "fabric of the universe" is the informational structure, and pancomputationalism (natural computationalism) is the idea that the universe is a computational system, which by physical processes "computes" its own next state. Based on this understanding, he advances the argument that paninformationalism and pancomputationalism, seen together as a paradigm for information, cognition and communication, are 
not enough to explain the role of the first-person conscious embodied social awareness. Arguing in the framework of cybersemiotics, Brier emphasizes importance of the production of signification and meaning, for which science currently lacks a detailed understanding in the framework of info-computationalism. Besides, he proposes a cybersemiotic theory of knowledge based on Peirce's semiotic framework as a step towards a more realistic theory of knowing, which is important for technological applications.

In Chapter 2, "Information Dynamics in a Categorical Setting", Mark Burgin builds a categorical models for information and information processes based on the general theory of information, which is a synthetic approach that organizes and encompasses all main directions in information theory. On the methodological level, the general theory of information is formulated as system of principles, explaining what information is and how to measure information. The goal of this chapter is to build a mathematical stratum of the general theory of information based on category theory. Abstract categories allow the author to develop flexible models for information and its flow, as well as for computers, networks and computation. In this context, portions of information are modeled by operators in system representation spaces.

There are two types of representation of information dynamics in categories: the categorical representation and functorial representation. The categorical representations of information dynamics preserve internal structures of information spaces associated with infological systems as their state or phase spaces, modeling portions of information by categorical information operators. The functorial representations of information dynamics preserve external structures of information spaces associated with infological systems as their state or phase spaces, modeling portions of information by functorial information operators. Properties of these types of representations are studied. In particular, sequential, concurrent and parallel compositions of categorical information operators are defined and analyzed. Obtained results facilitate building a common framework for information and computation. Now category theory is also used as a unifying framework for physics, biology, topology, and logic, as well as for the whole mathematics. This provides a base for analyzing physical and information systems and processes by 
means of categorical structures and methods. In addition, categorical models of computation are discussed, demonstrating relations between information and computation on the level of their mathematical models in the categorical setting. In Conclusion, the author formulates various open problems in the mathematical theory of information.

Chapter 3, "Mathematics as a biological process", presents Greg Chaitin's comparative analysis of complexity in mathematics, physics and biology. Complexity is one the main characteristics of any process (cf., for example, (Chaitin, 1987; Li and Vitanyi, 1997; Burgin, 2005)). This characteristic discriminates simple processes from complex ones and shows that processes in living organisms are especially complex. In contrast to this, Chaitin starts his chapter by pointing out that his algorithmic complexity does not apply to biology. On the other hand, he conjectures that the idea of complexity that comes from biology can be used to find the limits of mathematics. As an example, Chaitin describes Hilbert's program aimed at complete mechanization and automatization of mathematics. As we know, Gödel demonstrated that no finite set of axioms can allow derivation of all true sentences even in such a basic system as the formal (Peano) arithmetic. In essence, any finite set of axioms for arithmetic is either incomplete or inconsistent. So, the Hilbert program was to stay unfinished. Incompleteness discovered by Gödel is also reflected in the work of Turing (1936), who showed that there are basic things in mathematics which cannot be computed. Chaitin explains incompleteness and incomputability using the notion of algorithmic complexity. In algorithmic complexity, something has $N$ bits of complexity if you need an $N$-bit program to calculate it, irrespective how long this $N$-bit program takes to run. Unlike physics, biology is fundamentally complex and biological processes cannot be explained in simple equations. According to Chaitin, pure mathematics is even worse than biology when it comes to complexity, as it is provably infinitely complex.

Chaitin argues that the bits of the number $\Omega$ give maximally unknowable information in pure mathematics. This might be true if mathematicians would acquire information only by logical deduction. Applying more powerful methods, such as induction, mathematics can go beyond the number $\Omega$ (cf. (Burgin, 2005)). It is also necessary to understand that $\Omega$ is not a number in the conventional sense because it 
depends on the choice of a universal Turing machine or universal partial recursive function.

Even though biology is enormously complex, its algorithmic complexity, according to Chaitin, is finite, while pure mathematics has infinite algorithmic complexity. Gödel alleged that his theorems did not affect human mathematicians because humans have a "divine spark which enables them to directly intuit mathematical truth" helping them to decide undecidable problems. Turing, on the other hand, thought humans are not basically different from machines. Chaitin himself believes that people might be a mix of both. The connection between computation, information and biology is a recurrent theme in this chapter.

In Chapter 4, "Information, Causation and Computation", John Collier, starts with two basic assumptions:

1. All information takes a physical form.

2. Everything physical can be explained in dynamical terms.

This allows Collier to infer that information must be explicable in terms of forces and flows. As forces and flows are physical phenomena and physics can be expressed in terms of information (cf., for example, (Frieden, 1998) or (von Weizsäcker, 1985; Lyre, 2002)), this approach looks like information reverse engineering. Collier's explication is done in order to study causation and is based on Bateson's definition of information as the difference (Bateson, 1972). The chapter shows that bringing together causation (which makes a dynamical difference) with the logic of information flow makes possible to see causation as a sort of computation. This view is compatible with natural computationalism which sees the whole of the universe as a computing physical system.

In Chapter 5, "From Descartes to Turing: The Computational Content of Supervenience", Barry Cooper uses the notion of definability in a structure and the concept of a Turing machine with an oracle as a model of interactive computation to address the problem of supervenience of mental properties on physical ones. According to Kim (1993), supervenience represents the idea that mentality is, at the bottom, physically based, and there is no free-floating mentality unanchored in the physical nature of objects and events in which it is manifested. 
It is necessary to remark that a Turing machine with an oracle treated by Cooper as a model of interactive computation is as interactive as a simple a Turing machine introduced in 1936 (Turing, 1936) where there is interaction between the head and tape of the machine. In essence any computation is interactive when the computing device receives input and gives output. The notions of input and output imply interaction with the environment. However, the first explicitly interactive model of computation was neural network, the simplest case of which appeared in the work of McCulloch and Pitts (1943) being a precursor of nowadays connectionism.

Cooper also shows how to give a computational content to emergence, use emergence to link functions of the brain to higher mental activity, and apply definability to recursions involving mental representations. This leads to presenting the organism with a coherence and organic unity.

Chapter 6 presents "A Dialogue Concerning Two World Systems, Info-Computational Vs. Mechanistic." In it Gordana Dodig-Crnkovic and Vincent Müller compare the classical mechanistic world view, where Church-Turing thesis takes Turing Machines to be capable of modeling all possible computations, with the new emerging info-computationalism which takes the dynamics of the universe to be a process of computation, be it digital or analog, symbolic or sub-symbolic, technological or of the type known as natural computation (cf., for example (Ballard, 1997; MacLennan, 1999)). The argument is advanced so that for the first time in history of science, a scientific framework has a grounded capacity of embracing the biotic (living) world into the domain of computer science, and in particular, including the ability to rekindle the computational models of mind, based on natural computing. Of course, if the science is to remain "objective", which may be interpreted as "inter-subjective", this new understanding of life and mind would also have to be the third person view, comprising knowledge of what happens in the world when a human or an animal has conscious experience. In this context, the subjective experience, such as qualia, must be addressed by other scholarly, intellectual and artistic fields.

In Chapter 7, "Does Computing Embrace Self-Organization?", Wolfgang Hofkirchner analyzes the rise of the computer as man-made machine for processing information, the spread of PCs, the diffusion of 
ICTs and the penetration of social life including the natural environment as well as human bodies with "intelligent" devices. The author explores possibilities of computation and its relations to information and selforganization. It is demonstrated what happens if computers and computation would be treated from the position of technically oriented, mechanistic thinking. In this case, it is easy to show that computing in this restricted sense does not encompass self-organization.

The mechanistic way of thinking about computation brings researchers to the statements that "information generation defies being formalized, expressed by a mathematical function, or carried out by a computer" and "the generation of information escapes algorithmization, in principle." In reality, there is a big and flourishing area called algorithmic information theory, which is based on algorithmic, and thus, computational approach and which successfully studies information (cf., for example, (Chaitin, 1987; Li and Vitanyi, 1997; Burgin, 2005)).

In the chapter, mechanistic computation is also contrasted to emergence. At the same time, existing computational processes, which are much broader than their mechanistic counterpart, include a big and flourishing area called evolutionary computations (cf., for example, (Koza, 1992; Fogel, 1995; Michalewicz, 1996; Eberbach, 2005; Burgin and Eberbach, 2008; Burgin and Eberbach, 2009)). The basic idea of evolutionary computations is computational emergence in a form of evolution. One of the examples of such emergence is the Game of Life (cf., (Langton, 1989)).

Besides, theory of algorithms and computation has models, such as non-deterministic and probabilistic finite automata and non-deterministic and probabilistic Turing machines, which perform computations, but go far beyond strictly mechanistic procedures (cf., for example, (Hromkovic, 2005; Burgin, 2005)).

This demonstrates that the understanding of computing and information processing depends on the made assumptions, serving as litmus test, which indicates whether or not this understanding amounts to a really adequate paradigm.

In Chapter 8, "Analysis of Information and Computation in Physics Explains Cognitive Paradigms: From Full Cognition to Laplace Determinism to Statistical Determinism to Modern Approach", Vladik 
Kreinovich, Roberto Araiza, and Juan Ferret analyze the problem of prediction in physics from the computational viewpoint. They explicate the three main physical paradigms in understanding physical reality: Laplace determinism, statistical determinism, and information accessibility, which they call the Modern Approach. Laplace determinism means that knowing the initial conditions, it is possible to predict future behavior of the system that is observed. Statistical determinism means that knowing the initial conditions, it is possible to predict probabilities of different future events in the system that is observed. Information accessibility (Modern Approach) means that knowing the initial conditions, it is possible to predict the system behavior that is observed.

The authors show how these paradigms can be naturally explained by their computational analysis. To achieve this goal, they use concepts from the algorithmic information theory, such as Kolmogorov complexity, which was introduced by Ray Solomonoff, Andrey Kolmogorov and Gregory Chaitin, and algorithmic randomness (cf., for example, (Chaitin, 1987; Li and Vitanyi, 1997; Burgin, 2005)), as well as the novel, more physics-oriented versions of these concepts developed by the authors.

Chapter 9, "Bodies - Both Informed and Transformed Embodied Computation and Information Processing," by Bruce MacLennan is dedicated to an analysis of embodied computing. It emphasizes the importance of the development of physical computing or "embodied computing" for the future of computational technology, including the control of processes at nano-scales.

MacLennan observes the essential interrelationships between information processing and physical processes and discusses relationships between function, structure, regulation, causation, and the definition of computation. The chapter concludes with the claim that in order to fully exploit embodied computation, we need robust and powerful theoretical tools, but according to MacLennan the theory of Church-Turing computation, performed by an abstract logical machine is not suitable for the study of embodied computing. It is possible to add that by its essence, embodied computing is super-recursive and thus, its theory is a part (subtheory) of the theory of super-recursive algorithms (Burgin, 2005). 
In Chapter 10, "Computation on Information, Meaning and Representations: An Evolutionary Approach", Christophe Menant interprets computation as "a process of the dynamic change of information," which does not independently exist but is generated by the system that uses it for a definite purpose. This is in a good agreement with the general theory of information, where computation is regarded as information transformation, while information is understood as potency for changes (Burgin, 2003; 2005). According to the author, "information can be meaningless like thunderstorm noise or it can be meaningful like an alert signal. However, even thunderstorm noise participates to the generation of meaningful information about coming rain. Thus, it becomes important to analyze the relations between information, its meaning and representation. To make these relations explicit and constructive, the author uses the concept of the Meaning Generator System (MGS), which connects systems and their environment.

In Chapter 11, "Interior Grounding, Reflection, and SelfConsciousness, “ Marvin Minsky explores the problem of consciousness, emphasizing the view on the mind as a multi-level system of processes. He describes how researchers recognize consciousness and what might be happening when a person thinks consciously. The next question of interest is about how our mental levels develop and grow. Minsky criticizes the popular view of "grounding in experience" when the mental development is assumed to proceed by beginning with learning low-level reactions, waiting for each stage to consolidate before people can learn to think more abstractly. Subjective experience, ever so often emphasized by philosophers, is nothing really direct, but a complex process based on the " $n$-th hand reports" that have gone through many kinds of transformations. For example the visual system in a human brain includes dozens of different processing centers. Sensations are complex reflective activities, sometimes involving cascades in which parts of the brain are affected by signals whose origins we cannot detect and thus hard to explain. In agreement with Kant (cf., (Kant, 1902-1956)), Minsky states that our empirical knowledge is a combination of that which we receive through sensory impressions (experience), and knowledge which the cognition supplies from within, by different mechanisms like associations, reasoning, analogical thinking, and similar. This brings 
Minsky to the problem of knowledge representation. In contrast to the most popular approaches where researchers advocate one form of knowledge representation, e.g., logic or semantic networks, Minsky argues that knowledge in the brain forms a hierarchy of different levels of representation, from micronemes, neural networks, $k$-lines and $k$-trees, semantic networks, frames, picture frames, trans-frames to narrative stories - each of the levels having specific dedicated role in knowledge representation. Methodological analysis of knowledge also supports this conjecture, separating three basic levels of knowledge: the micro-level, macro-level and mega-level (cf., (Burgin, 2010)). Each of these levels comprises several specific forms of knowledge representation.

In Chapter 12, “A Molecular Dynamic Network: Minimal Properties and Evolutionary Implications," Walter Riofrio is proposing a hypothesis addressing the problem of the emergence and self-maintenance of protocellular organization, describing a kind of evolutionary mechanism before the life as we understand it today appeared. Riofrio builds his approach on the observation that for biological systems, robustness and evolvability are in continuous tension.

In order to understand the basic properties of life, it is crucial to understand the first forms that developed much earlier than previously thought. This chapter indicates that the development of interconnected molecular processes is possible evolving from random initial conditions.

In Chapter 13, "Super-recursive Features of Evolutionary Processes and the Models for Computational Evolution," Darko Roglic is developing a computational approach based on biological information processing, computational evolution in the sense of (Banzhaf, et.al., 2006), and inductive Turing machines, a computational model introduced by Burgin and approved by Kolmogorov (Burgin, 1983). The goal is using ideas that reflect natural biological system functioning, to develop new kinds of computational evolutionary systems. Roglic describes and analyzes information processing in biological systems, as well as computation by biologically oriented computers. As the author writes, to build systems that can adapt to their environments and learn from their experience is the long-standing goal, attracting researchers from many fields. Giving an overview of biological information processing, Roglic comes to a grounded conclusion that even evolvable capabilities of bacteria for 
adaptation in rapidly changing and extremely hostile environment are not achievable for modern computational systems working in the paradigm of the Church-Turing thesis. To overcome limitations of recursive algorithms working in the boundaries of the Church-Turing thesis, super-recursive evolutionary information processing is used.

In addition, conventional Turing machines cannot change their instructions (rules of computation) in the process of computation because their instructions are separated from the processed data. Discussing ideas of a learning machine, Turing (1950) insisted that the rules of computation have to be time-invariant. At the same time, self-adaptation demands changing the rules. This brings Roglic to the idea of selfmodification in computing systems.

Reflexive Turing machine was suggested by Burgin (1992) as a generic model for programs or automata that change (improve) themselves while they are working. Kleene (1960) formulated a conjecture that an algorithm that changes (improves) itself while working can have higher computational/decision power that Turing machines. In (Burgin, 1992), it is proved that a Turing machine can simulate any reflexive Turing machine. This disproved the Kleene conjecture and gave more evidence in support of the Church-Turing Thesis, although at that time it was known (Burgin, 1987) that the Church-Turing Thesis in its strong form (equating all possible computations with Turing machines) is invalid.

However, it was proved (Burgin, 1993) that reflexive Turing machines can be much more efficient than Turing machines, essentially reducing time of computation. This result supports the approach of Roglic who uses ideas from natural biological systems in developing new kinds of computational evolution systems. To this end, Roglic describes a EC machine, bacteria-like EC(BL-EC) machine and Sequencer machine as four-dimensional patterns of horizontal-vertical computation, which reflects the structure, functioning and ontogenetic development of a living organism. BL-EC machine has two basic modules: Interactor program as a part of input and output information processing system and Processor as a part of working information processing system.

In Chapter 14, "Towards a Modeling View of Computing," Oron Shagrir presents a general characterization of a computing system, building on the observation that computation operates with information 
or over representations that does not require structural constraint in a sense of an algorithmic or rule-governed process. Shagrir provides an example from computational neuroscience: the modeling account of computing of the oculomotor integrator, which models certain mathematical relations "in the world" by the mathematical relations defined for the cellular electric activity. In general, there is an "isomorphism" between the formal or mathematical relations that represent actual relations between the states of the modeled system, and mathematical relations between the states of the modeling system. The idea of computation as modeling can be traced back to analog computers.

This correspondence shows that modeling knowledge has the form of the basic element of knowledge described in (Burgin, 2010). This modeling knowledge is represented by the following diagram

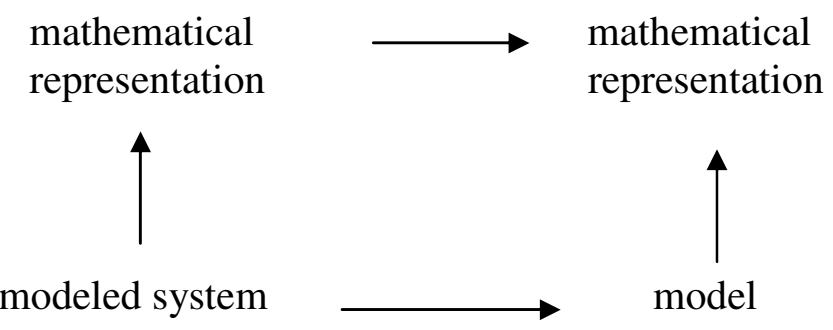

Shagrir provides a general characterization of a computing system, which is both semantic and formal. The semantic feature consists of information-processing, which maps one set of representations to another, while the formality refers to functional relations between the ccomputing system states, being similar to the mathematical relations between the entities that are represented.

In Chapter 15, "What's Information, for an Organism or Intelligent Machine? How Can a Machine or Organism Mean?", Aaron Sloman studies problems of information and meaning with the emphasis on clarifying the concept of information, providing a profound methodological analysis of the current situation. Giving reasonable arguments, he asserts that the concept "information" cannot be defined explicitly in terms of simpler concepts, including "matter" and "energy". Instead, the meanings of such basic phenomena are defined implicitly in part by the structure of the theories in which they occur, and in part by 
the way those theories are used in practice. Sloman gives persuasive evidence and explains why it is impossible to define information without circularity in such a way that the definition would encompass diverse uses and interpretations of the term information. Several other researchers share this opinion (cf., for example, (Capurro, et al, 1999; Melik-Gaikazyan, 1997)).

At the same time, a comprehensive definition of information in the general theory of information (Burgin, 2010) does not have circularity and encompasses a host of diverse definitions of information, either making them specializations of the general definition or assigning a more adequate meaning to those definitions. For instance, information entropy $H(m)$ is often called Shannon's information. However, as it is explained in the general theory of information, $H(m)$ is not information but a measure of information in the message $m$.

It looks like a paradox: several authoritative researchers give valid arguments for impossibility to elaborate a concise definition of information, while such a definition already exists. However, there is no contradiction in this situation. Researchers reject a possibility of a conventional definition of information, while in the general theory of information the definition is parametric, depending on a system parameter. Variations of this parameter allow one to obtain the diversity of all meanings and interpretations (sometimes in a more adequate form) of the term information.

However, to understand and use more efficiently information and information processes, it is necessary to specify different kinds and types of information, describing essential properties of the studied phenomena. To do this, Sloman elucidates and studies three basic aspects of information: content, function (or use, or causal role) and the medium (or representation, or carrier) in which information is expressed. It is possible for the same information content to be put to different uses, to be conveyed by different media and to play different roles. Thus, when information is used, it is natural to distinguish the content of the information, which Sloman calls phrastic following (Hare, 1952), from the use that is being made of it, which Sloman calls neustic also following (Hare, 1952). Besides, as Sloman rightly notes, from the earliest days of AI and software engineering, it was clear that choice of information 
representation could make a large difference to the success of a particular information processing system. This perfectly correlates with the basic principles of the general theory of information (Burgin, 2010).

The main goal of Chapter 16, "Inconsistent Knowledge as a Natural Phenomenon", by Kees de Vey Mestdagh and Jaap Henk Hoepman is ranking of reasonable inferences as a computational approach to naturally inconsistent (legal) theories. At first, the authors give a broad perspective on problems of inconsistent knowledge and inconsistent information, concentrating on the natural inconsistency, which results from the distributed character of information processing and variation between information content in different carriers of information. The bounded character of information and information processing allows for natural inconsistency (in the sense of inconsistency being the result of the different perspectives as opposed to faulty perception or other forms of faulty processing like processing on the basis of incomplete knowledge). Natural inconsistency constitutes no general problem. It only generates a specific practical problem if a common perspective is needed. However, a common perspective is frequently required for a variety of reasons ranging from the demand for making a decision (on a common perspective or action) to the need for a decidable logic or for a finite algorithm. There are three main approaches to solve the problem of common perspective: universalism, utilitarianism and contractarianism. Universalism claims that there are universal decisive principles (cf., for example, Kant's categorical imperative (Kant, 1902-1956)). Utilitarism promoted by philosophers, such as David Hume, Jeremy Bentham, John Stuart Mill, claims that it is possible to make a decisive cost-benefit analysis (cf., for example, Kant's hypothetical imperative (Kant, 19021956)). Contractarianism avoids the semantic problems of utilitarism by introducing a purely formal decisive criterion (pacta sunt servanda). None of these approaches have ever been made computationally tractable. Inconsistency as a natural phenomenon explains why they never will. The core of the problem is that there is not only natural inconsistency at the level of perspectives on the actual situation, but also natural inconsistency at the level of the principles used to decide for a common perspective. There is no universal preferential ordering of perspectives at both levels, because there is no (known, let alone a 
universally recognized) universal processor and because there is no exhaustive or non contradictory set of universal or utilitarian principles or contracts available.

Each domain of knowledge is more or less affected by this problem, but the domain of legal knowledge is affected by it intensely, since it consists of the rules and procedures used to describe and to solve legal conflicts, that presuppose contradictory and hence inconsistent perspectives. An analysis of the solution of the problem of common perspective found in the legal domain can most likely be used to solve the problem in other domains.

Human processors of legal knowledge follow formal and informal problem solving methods to reduce the number of valid (legitimate) legal perspectives and eventually to decide, temporally and within a specific context, to a common perspective. The formal methods are based on universal properties of formally valid legal argument. The informal methods are based on legal heuristics consisting in tentative decisive legal principles.

The first category can be formalized by logic because it applies peremptory to all legal perspectives. The second category can not be fully formalized by logic because, although it applies commonly, it always can be refuted by a contradictory decisive principle and even by the mere existence of an underlying contradictory argument.

The authors develop the Logic of Reasonable Inferences that formalizes the reduction of all actual legal perspectives to all formally valid legal perspectives. This logic is a legal logical variety in the sense of (Burgin, 1995; 2004). In the process of the logic development, decisive legal principles are categorized into three classes. The properties of the three classes are used to define the semantics of meta-predicates, which can be used to rank the remaining perspectives computationally. This approach is very perspective because logical varieties represent the natural development of logical calculi and thus, they show the direction in which mathematical logic will inevitably go.

In the last part of the chapter, the authors illustrate the behavior of the Logic of Reasonable Inferences in combination with the meta-predicates by a complex legal example. 
In Chapter 17, "On the Algorithmic Nature of the World", Hector Zenil and Jean-Paul Delahaye try to identify evidence in support of or in contravention of the claim that the world is algorithmic in nature. The idea that nature functions exclusively in the form of computation has been taken increasingly seriously following the lead of some authors, such as Fredkin, Schmidhuber, and Wolfram. However, any scientific hypothesis has to be verified by experiments and observation. In contrast to this, there has been no way to either verify or refute the idea of the algorithmic nature of the world. The authors describe a test for this hypothesis based on mathematical statistics, the theory of algorithmic (Kolmogorov) complexity and an experimental evaluation of Levin's universal distribution. To achieve their goal, Zenil and Delahaye perform statistical comparisons of the frequency distributions of data samples from physical sources, such as repositories of information in the form of images from the web, data stored in a hard drive, computer programs and DNA sequences, and the output frequency distributions generated by purely algorithmic means, e.g., by running abstract computing machines, such as Turing machines, cellular automata and Post Tag systems. The authors find some indications of correlations having weak to strong significance. This is the case with distributions from the chosen abstract devices, as well as with data from the chosen physical sources. Each of these sources separately turned out to show several degrees of correlation. At the same time, while the correlation between the two sets is partial, each distribution is correlated with, at least, one other distribution produced by an abstract model of computation.

To conclude, it is our hope that the research presented in this volume will bring us a significant step closer to understanding the phenomena of information and computation and their relationships, making a difference that makes a difference. Obviously, at this stage we do not want to claim that we have a "final theory of everything" in front of us (even if such a theory would be possible), but the ideas developed and results obtained in this book provide a grounded proof of deep and profoundly important research going on in the foundations of information and computation.

Last but not least we want to express our sincere gratitude to the authors both for their highly interesting chapters, each of them addressing an important topic for the future development of the research 
field, and for their contributions in forming the book as a whole. This book presents a substantially collaborative project, where authors even helped in the reviewing process, taken part in discussing topics and contributed with suggestions about the way of presentation of the book. We would also like to thank Kaj Børge Hansen, Patrick Allo, Luciano Floridi and Ruth Hagengruber for reviewing parts of the book.

\section{References}

Adriaans, P. and van Benthem, J. (Eds) (2008) Handbook on the Philosophy of Information, North Holland

Ballard, D.H. (1997) An Introduction to Natural Computation, MIT Press, Cambridge

Banerjee, P. Technology critical to turning mass of data into useful insight, FT.com, April 8,2009

Banzhaf, W.,Beslon, G., Christensen, S., Foster, A.J, Kepes, F., Lefort, V., Miller, F.J., Radman, M. and Ramsden, J. (2006) Guidelines: From artificial evolution to computational evolution: a research agenda, Nature Reviews Genetics, v. 7, pp. 729-741

Bateson, G. (1972) Steps to an Ecology of Mind, Ballantine Books, New York

Boisot, M. and Canals, A. (2004) Data, Information, and Knowledge: Have we got it right, Journal of Evolutionary Economics, v. 14, pp. 43-67

Bournez, O. (1999) Achilles and the tortoise climbing up the hyper-arithmetical hierarchy, Theoretical Computer Science, v. 210, No.1, pp. 21-71

Burgin, M. S. (1983) Inductive Turing Machines, Notices of the Academy of Sciences of the USSR, v. 270, No. 6, pp. 1289-1293 (translated from Russian, v. 27, No. 3 )

Burgin, M. (1987) The Notion of Algorithm and the Turing-Church Thesis, In Proceedings of the VIII International Congress on Logic, Methodology and Philosophy of Science, Moscow, v. 5, part 1, pp. 138-140

Burgin, M.S. (1992) Reflexive Calculi and Logic of Expert Systems, in Creative processes modeling by means of knowledge bases, Sofia, pp. 139-160

Burgin, M. (1993) Reflexive Turing Machines and Calculi, Vychislitelnyye Sistemy (Logical Methods in Computer Science), No. 148, pp. 94-116, 175-176

(in Russian)

Burgin, M. (1995) Logical Tools for Inconsistent Knowledge Systems, Information: Theories \& Applications, v.3, No. 10, pp.13-18

Burgin, M. (1997) Information Algebras, Control Systems and Machines, No.6, pp. 5-16 (in Russian)

Burgin, M. (2001) Information in the Context of Education, Journal of Interdisciplinary Studies, v. 14, pp. 155-166

Burgin, M. (2003) Information Theory: A Multifaceted Model of Information, Entropy, v. 5 , No. 2, pp. 146-160

Burgin, M. (2004) Logical Tools for Program Integration and Interoperability, in Proceedings of the IASTED International Conference on Software Engineering and Applications, MIT, Cambridge, pp. 743-748 
Burgin, M. (2005) Super-recursive Algorithms, Springer, New York/Heidelberg/Berlin Burgin, M. (2005a) Is Information Some Kind of Data? Proceedings of the Third Conference on the Foundations of Information Science (FIS 2005), Paris, France, July, pp. 1-31 (electronic edition: http://www.mdpi.net/fis2005/proceedings.html)

Burgin, M. (2010) Theory of Information: Fundamentality, Diversity and Unification, World Scientific, New York/London/Singapore

Burgin, M. and Eberbach, E. (2008) Cooperative Combinatorial Optimization: Evolutionary Computation Case Study, BioSystems, v. 91, no.1, pp. 34-50

Burgin, M. and Eberbach, E. (2009) On Foundations of Evolutionary Computation: An Evolutionary Automata Approach, in Handbook of Research on Artificial Immune Systems and Natural Computing: Applying Complex Adaptive Technologies, Section II: Natural Computing, Section II.1: Evolutionary Computing, Chapter XVI, Medical Information Science Reference/IGI Global, Hershey, New York, pp. 342-360

Capuro, R., and Hjorland, B. (2003) The Concept of Information, Annual Review of Information Science and Technology, v. 37, No. 8, pp. 343-411

Capurro, R., Fleissner, P., and Hofkirchner, W. (1999) Is a Unified Theory of Information Feasible? In The Quest for a unified theory of information, Proceedings of the $2^{\text {nd }}$ International Conference on the Foundations of Information Science, pp. 9-30

Castell, L., Drieschner, M., and von Weizsäcker, C.F. (Eds.) (1975-1986) Quantum Theory and the Structures of Time and Space (6 volumes), Hanser, Munich

Chaitin, G.J. (1987) Algorithmic Information Theory, Cambridge University Press, Cambridge

Dodig-Crnkovic, G. (2006) Investigations into Information Semantics and Ethics of Computing, Mälardalen University Press (http://urn.kb.se/resolve?urn=urn:nbn:se:mdh: diva-153)

Dodig-Crnkovic, G. (2009) Information and Computation Nets. Investigations into Infocomputational World, VDM Verlag

Eberbach, E. (2005) Toward a theory of evolutionary computation, BioSystems, v. 82, pp. $1-19$

Frieden, R.B. (1998) Physics from Fisher Information, Cambridge University Press, Cambridge

Fricke, M. (2008) The Knowledge Pyramid: A Critique of the DIKW Hierarchy, Preprint (electronic edition: http://dlist.sir.arizona.edu/2327/)

Fogel D.B. (1995), Evolutionary Computation: Toward a New Philosophy of Machine Intelligence, IEEE Press

Gupta, V., Jagadeesan, R., and Saraswat, V.A. (1999) Computing with Continuous Change, Science of Computer programming, v. 30, No. 1/2, pp. 3-49

Hare, R. (1952) The language of Morals, Oxford University Press, Oxford

Hromkovic, J. (2005) Design and Analysis of Randomized Algorithms, Springer, New York

Kant, E. (1902-1956) Gessamelte Schriften, Der Preussischen Akademie der Wissenschaften, de Gruyter, Berlin/New York

Kelemen, J. (2006) On a Possible Future of Computationalism, 7th International Symposium of Hungarian Researchers on Computational Intelligence, pp. 51-56

Kim, J. (1993) Mind and Supervenience, Cambridge University Press, Cambridge 
Kleene, S.C. Mathematical logic: constructive and non-constructive operations, in "Proceedings of the International Congress of Mathematicians, 1958", Cambridge University Press, New York, 1960, pp. 137-153

Kogut, B. and Zander, U. (1992) Knowledge of the Firm: Combinative Capabilities, and the Replication of Technology, Organization Science, v. 3, No. 3, pp. 383-397

Koza, J. (1992) Genetic Programming, I, II, III, MIT Press, 1992, 1994, 1999

Langton, C. G. (1989) Artificial Life, in Artificial Life, pp. 1-47

Li, M., and Vitanyi, P. (1997) An Introduction to Kolmogorov Complexity and its Applications, Springer-Verlag, New York

Lyre, H. (2002) Quanten der Informationtheorie: Eine philosophischnaturwissenschaftliche Einfürung, Fink, Munich

Machlup, F. and Mansfield, U. (Eds.) (1983) The Study of Information: Interdisciplinary Messages, Wiley, New York

MacLennan, B.J. (1999) Field computation in natural and artificial intelligence, Information Sciences, v. 119, pp. 73-89

McCulloch, W. S., and Pitts, E. (1943) A Logical Calculus of the Ideas Immanent in Nervous Activity, Bulletin of Mathematical Biophysics, v. 5, pp.115-133

Meadow, C. T. and Yuan, W. (1997) Measuring the impact of information: Defining the concepts, Information Processing and Management, v. 33, No. 6, pp. 697-714

Melik-Gaikazyan, I. V. (1997) Information processes and reality, Nauka, Moscow (in Russian, English summary)

Michalewicz, Z. (1996) Genetic Algorithms + Data Structures = Evolution Programs, Third edition, Springer-Verlag

Moore, C. (1996) Recursion Theory on the Reals and Continuous-time Computation: Real numbers and computers, Theoretical Computer Science, v. 162, No. 1, pp. 23-44

Shannon, C. (1941) Mathematical Theory of the Differential Analyzer, J. Math. Physics, MIT, v. 20, pp. 337-354

Stenmark, D. (2002) The Relationship between Information and Knowledge and the Role of intranets in Knowledge Management, in Proceedings of the 35th Annual Hawaii International Conference on System Sciences (HICSS-35), v. 4, IEEE Press, Hawaii (http://csdl2.computer.org/comp/proceedings/hicss/2002/1435/04/14350104b.pdf)

Tuomi, I. (1999) Data is More Than Knowledge: Implications of the Reversed Knowledge Hierarchy for Knowledge Management and Organizational Memory, Journal of Management Information Systems, Vol. 16, No. 3, pp. 107-121

Umpleby, S.A. (2007) Physical relationships among matter, energy and information, Systems Research and Behavioral Science Systems Research, v. 24, pp. 369-372

von Weizsäcker, C.F. (1985) Aufbau der Physik, Hanser, Munich, Germany (Eglish translation: The Structure of Physics, Springer, Berlin/Heidelberg/New York, 2006)

von Weizsäcker, C.F., Scheibe, E., and Süssmann, G. (1958) Komplementarität und Logik, III (Mehrfache Quantelung), Zeitschrift für Naturforschung, v. 13, pp. 705-721 